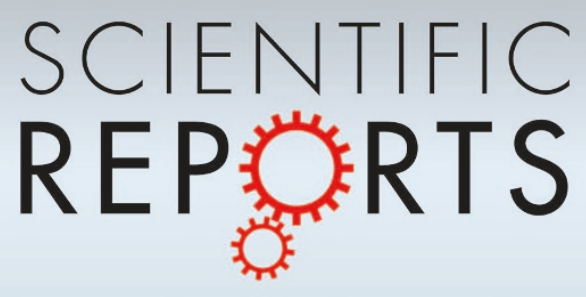

OPEN

SUBJECT AREAS:

PHASE TRANSITIONS

AND CRITICAL

PHENOMENA

MAGNETIC PROPERTIES AND

MATERIALS

Received

27 December 2013

Accepted

29 May 2014

Published

20 June 2014

Correspondence and requests for materials should be addressed to

W.M.L. (wliu@iphy. ac.cn)

\section{Layer Anti-Ferromagnetism on Bilayer Honeycomb Lattice}

\author{
Hong-Shuai Tao, Yao-Hua Chen, Heng-Fu Lin, Hai-Di Liu \& Wu-Ming Liu
}

Beijing National Laboratory for Condensed Matter Physics, Institute of Physics, Chinese Academy of Sciences, Beijing 100190, China.

Bilayer honeycomb lattice, with inter-layer tunneling energy, has a parabolic dispersion relation, and the inter-layer hopping can cause the charge imbalance between two sublattices. Here, we investigate the metal-insulator and magnetic phase transitions on the strongly correlated bilayer honeycomb lattice by cellular dynamical mean-field theory combined with continuous time quantum Monte Carlo method. The procedures of magnetic spontaneous symmetry breaking on dimer and non-dimer sites are different, causing a novel phase transition between normal anti-ferromagnet and layer anti-ferromagnet. The whole phase diagrams about the magnetism, temperature, interaction and inter-layer hopping are obtained. Finally, we propose an experimental protocol to observe these phenomena in future optical lattice experiments.

T

he bilayer honeycomb lattice (BHL) has attracted enormous interest in both experimental and theoretical research. Lots of novel phenomena have been found in BHL, for instance, the quantum Hall effect, quantum spin Hall effect, and chiral superconductivity ${ }^{1-11}$. However, the real charge and magnetic order induced by Coulomb interaction are still challenges in the strongly correlated $\mathrm{BHL}^{12-16}$. A quadratic dispersion relation, signed by two touching bands in the corners of Brillouin zone, can be found in BHL, which is driven by the interlayer hopping. In addition, the spontaneous symmetry can be broken by the dimers when the inter-layer hopping changes. Some amazing phases may emerge, such as layer anti-ferromagnetic phase and paramagnetic insulator phase. Previous work mainly focus on the electronic properties of $\mathrm{BHL}^{17-24}$, and the phase diagram for the magnetic phase transition induced by the interaction and dimers are still absent. Moreover, the progress of optical lattice provides us a useful tool to set a controllable and clearness experimental platform to simulate the strongly correlated BHL, in which the interaction between trapped fermionic cold atoms can be tuned by the Feshbach resonance $e^{25-31}$.

The dynamical mean-field theory (DMFT) has been proved to be a very useful and effective tool ${ }^{32-35}$, which has made significantly progress as in the study of metal-insulator phase transition. The DMFT is exact when investigating the strongly correlated system in the infinite-dimension, in which the self-energy is independent of momentum. However, in low-dimensional systems, the quantum fluctuation and short range correlations play important roles, which are ignored in DMFT. The cellular DMFT (CDMFT), as a cluster extension of DMFT, effectually incorporates the spatial correlations by mapping the many-body problem into finite clusters embedding in a self-consistent media ${ }^{36-39}$. In two-dimensional systems CDMFT is more precise than DMFT, and is more effective to investigate the phase transition in low and multi-component systems ${ }^{31,40,41}$.

In this report, we investigate the finite temperature metal-insulator and magnetic phase transition in strongly correlated bilayer honeycomb lattice (BHL) by combining the cellular dynamical mean-field theory (CDMFT) with continue-time quantum Monte Carlo (CTQMC) method ${ }^{42}$. A phase transition from paramagnetic phase to anti-ferromagnetic phase occurs by investigating the magnetization. In a proper value of inter-layer hopping, a novel layer anti-ferromagnetic phase emerges. The layer anti-ferromagnetic phase can be broken by the increasing inter-layer hopping, and a paramagnetic phase can be found. Moreover, the nonlocal inter-layer hopping plays an important role on localizing the free election and modifying the spatial distribution of the electron in lattice sites, especially in dimer sites. For instance, the dimer sites of our system are double occupied, and the nondimer sites are single occupied. We have also presented the DOS, double occupancy, and fermi surface below, which can be directly detected in future experiments.

\section{Results}

The strongly correlated bilayer honeycomb lattice. Fig. 1(a) shows the lattice structure of BHL. $A_{1}$ and $B_{1}$ denotes the sites on top-layer, while $A_{2}, B_{2}$ signs the bottom-layer sites. The inter-layer band connects $A_{1}$ (top) 
(a)

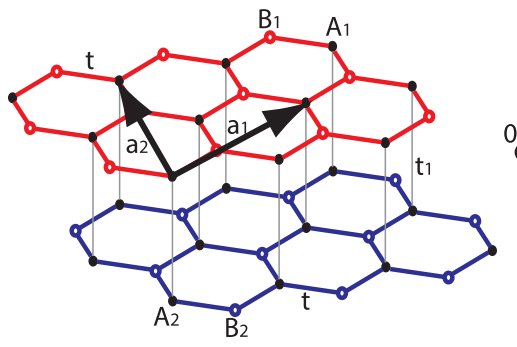

(b)

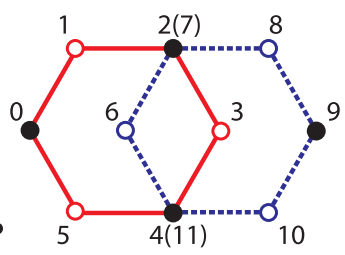

The cellular of the system
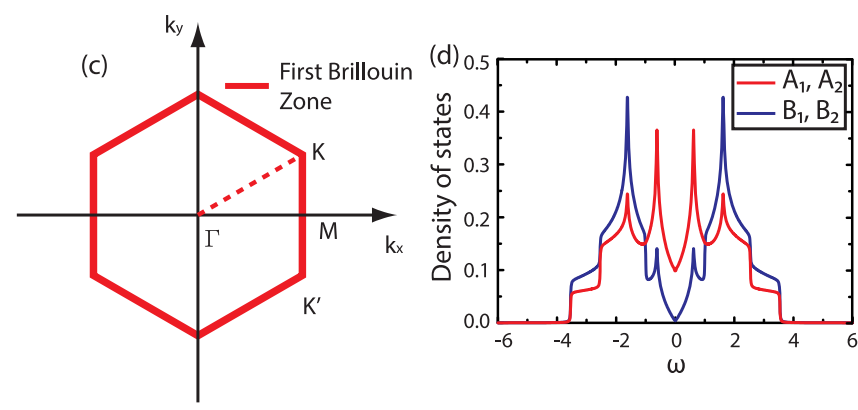

Figure 1 The structure of bilayer honeycomb lattice and its qualities in the non-interacting limit. (a): Bernal stacking of the bilayer honeycomb in real-space with intra- and inter-layer hopping $t$ and $t_{1}$ between the sublattice $A_{1}, B_{1}$ on top-layer and $A_{2}, B_{2}$ on bottom-layer. The black arrows $a_{1}$ and $a_{2}$ are the lattice vectors. (b): The cellular of our bilayer system in cellular dynamical mean-field theory (CDMFT). The red solid line containing sites $0,1,2,3,4,5$ belongs to top-layer and blue dotted line with sites $6,7,8,9,10,11$ belongs to bottom-layer. (c): Reciprocal lattice of bilayer honeycomb lattice. The thick red line shows the first Brillouin zone. The $\Gamma, K, M$ and $K^{\prime}$ points denote high symmetry points in first Brillouin zone. (d): Density of states of our system for $A_{1} / A_{2}$ and $B_{1} / B_{2}$ sites when $U$ $=0$.

and $A_{2}$ (bottom) sites. The first Brillouin zone can be found in Fig. 1(c), in which the $\Gamma, K, M$ and $K^{\prime}$ shows the points with different asymmetry in the $k$-space. The non-interaction density of states (DOS) for different sites when $t_{1}=t$ is shown in Fig. 1(d), which is different from the mono-layer honeycomb lattice ${ }^{43}$. The low-energy dispersion is quadratic, which is linear in the monolayer case. There is no band gap between the conduction and valence bands in BHL. In this report, we investigate the phase transition on the half-filling BHL. We consider the standard Hubbard model:

$$
H=-t \sum_{\langle i j\rangle \sigma \alpha}\left(c_{i \sigma \alpha}^{\dagger} c_{j \sigma \alpha}+\text { h.c. }\right)+U \sum_{i \alpha} n_{i \uparrow \alpha} n_{i \downarrow \alpha}-t_{1} \sum_{i \sigma \alpha} c_{i \sigma \alpha}^{\dagger} c_{i \sigma(1-\alpha)}-\mu \sum_{i \sigma \alpha} n_{i \sigma \alpha},
$$

where $t$ is the intra-layer nearest-neighbor hopping energy, $U$ is the Coulomb interaction, $t_{1}$ denotes the inter-layer hopping energy, $\mu$ is the chemical potential, which is adjusted to keep the system at halffilling, $c_{i \sigma \alpha}^{\dagger}$ and $c_{i \sigma \alpha}$ denote the creation and annihilation operators of fermionic atoms on site $i$ with spin $\sigma$ respectively, and $\alpha$ shows the layer parameter ( $\alpha=0$ means the top-layer, and $\alpha=1$ denotes the bottom-layer), $n_{i \sigma \alpha}=c_{i \sigma \alpha}^{\dagger} c_{i \sigma \alpha}$ corresponds to the density operator. Here we set $t=1$, which is also used as the energy unit in this report.

The metal-insulator phase transition. The $t_{1}-U$ phase diagram is shown in Fig. 2. The phase boundary of the metal and insulator is shown by the red solid line with square plots. When $t_{1}=0$ the critical point of metal-insulator phase transition is $U_{c}=4.7$, meaning that BHL degenerates into a mono-layer one. This result is the same as Ref. 16. In order to study the magnetic phase transition, we define the staggered magnetization as $M=\Sigma_{i} \operatorname{sgn}(i)\left(<n_{i \uparrow}>-<n_{i \downarrow}>\right)$, where $i$ denotes the lattice index, $\operatorname{sgn}(i)=1$ for $A_{1}$ and $A_{2}$ sites and $\operatorname{sgn}(i)=$

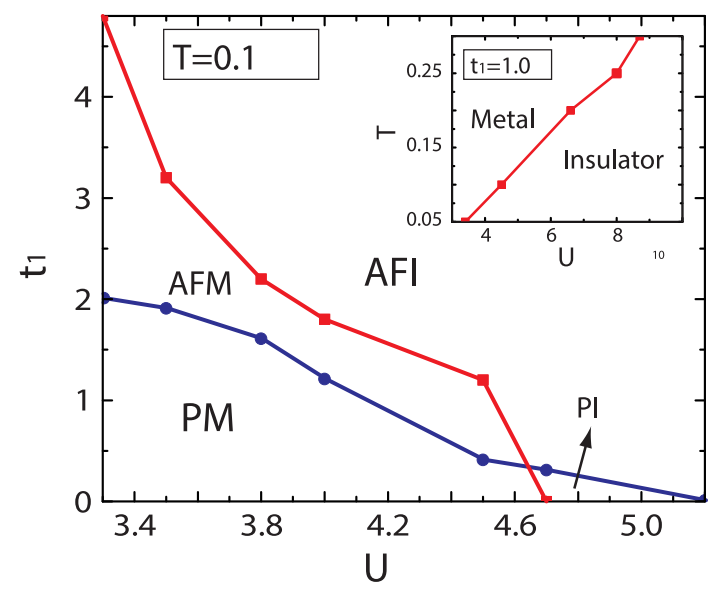

Figure $2 \mid$ Metal-insulator phase diagram in fixed $T$ or $t_{1}$. Phase diagram as a function of inter-layer hopping $t_{1}$ and interaction $U$ at $T=0.1$. The red solid line shows the boundary of metal-insulator phase transition, and the blue solid line denotes the magnetic phase transition, which divides the phase into paramagnetic metal (PM: $M=0$ and $\Delta E=0$ ), paramagnetic insulator (PI: $M=0$ and $\Delta E \neq 0$ ), anti-ferromagnetic metal (AFM: $M \neq 0$ and $\Delta E=0$ ) and anti-ferromagnetic insulator (AFI: $M \neq 0$ and $\Delta E \neq 0$ ). Inset: Phase diagram as a function of temperature $T$ and interaction $U$ at fixed $t_{1}$.

-1 for $B_{1}$ and $B_{2}$ sites. The phase boundary of non-magnetic state and magnetic state is shown by blue solid lines with circle points. As shown in Fig. 2, when $2<t_{1}<4.8$, anti-ferromagnet can be found. When $U$ is increased, a phase transition from gapless antiferromagnetic metal (AFM), where $\Delta E=0, M \neq 0$ and $\Delta E$ here is single particle gap, to gapped anti-ferromagnetic insulator (AFI), where $\Delta E \neq 0, M \neq 0$, occurs. When $t_{1}<2$, a paramagnetic metal (PM), where $\Delta E=0, M=0$, is formed, and a PM-AFM-AFI transition happens when $U$ is increased. In the value of $0<t_{1}<$ 0.2 , when $U>4.7$, a small region, which is named as paramagnetic insulator (PI) is found with $\Delta E \neq 0$ and $M=0$.

Double occupancy is always used to measure the localization of the electrons directly, which is an important parameter to observe the metal-insulator phase transition ${ }^{44}$. In this report, we investigate the double occupancy $D_{o c c}=\partial F / \partial U=\frac{1}{4} \Sigma_{i}<n_{i \uparrow} n_{i \downarrow}>$ as a function of

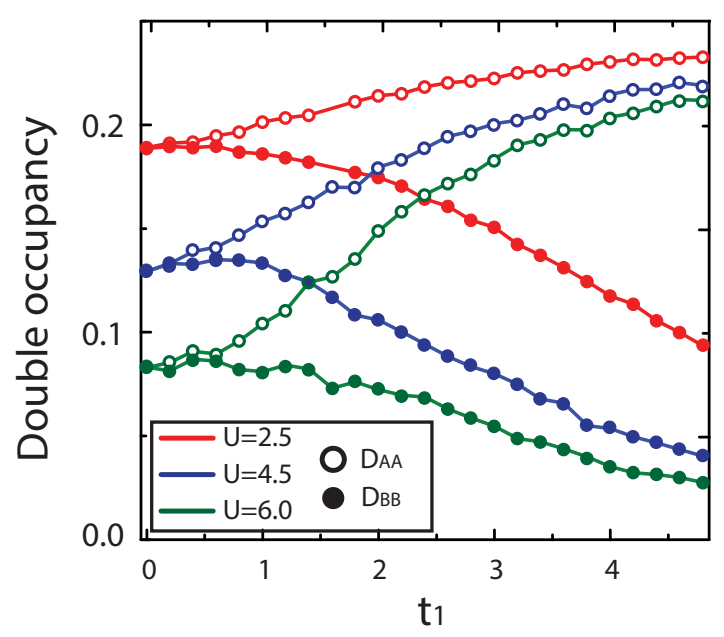

Figure 3 The evolution of double occupancy $D_{\text {occ }}$ The double occupancy as a function of inter-layer hopping $t_{1}$ for different interaction $U$ at temperature $T=0.1$. The dimer sites tend to be double occupied however non-dimer sites tend to be single occupied, with increasing $t_{1}$. 

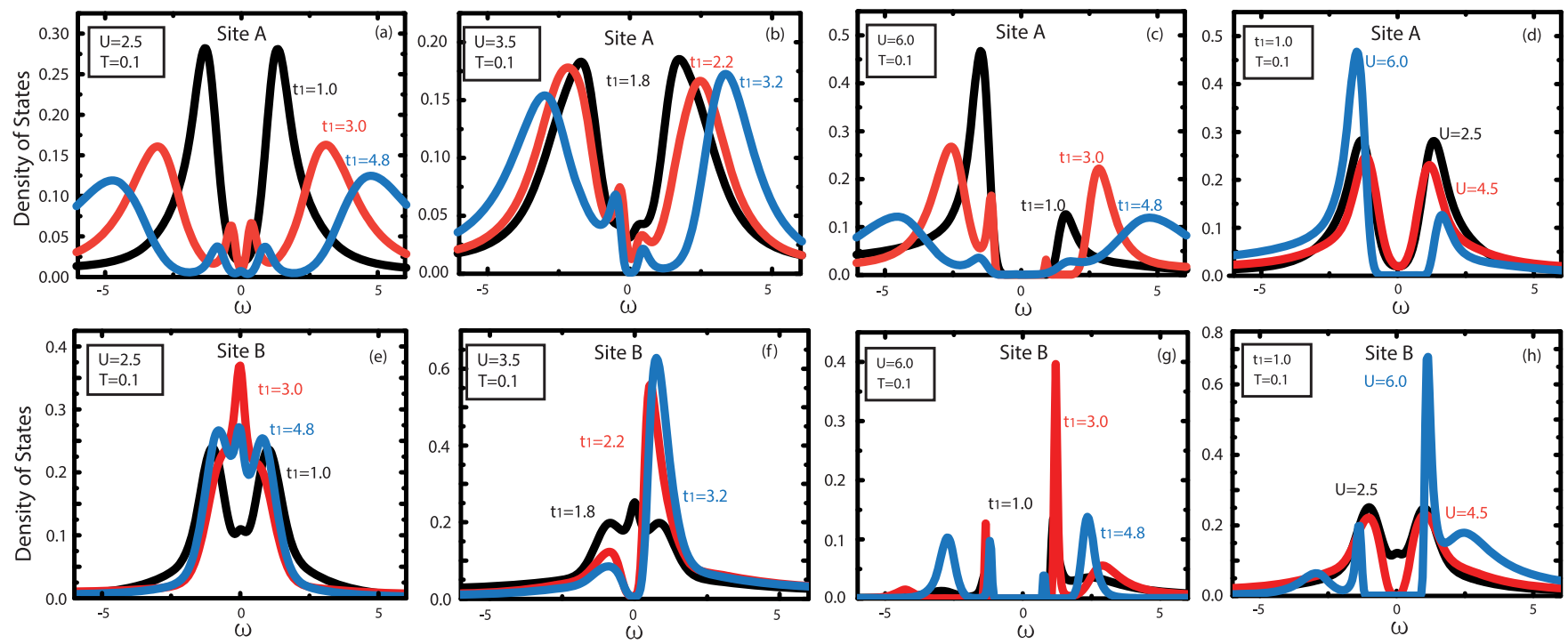

Figure $4 \mid$ The density of states. (a) - (c), (e) - (g): The density of states as a function of frequency $\omega$ for different inter-layer hopping $t_{1}$ at temperature $T$ $=0.1$. (a), (e): The metallic phase at $U=2.5$ for A sites and B sites. (b): For A sites, as we increase $t_{1}$, system undergoes a phase transition from metal (at $U=3.5$ and $t_{1}=1.8$ ) to insulator (at $t_{1}=3.2$ ). Single particle excitation gap opens at about $t_{1}=3.2$. (f): For B sites, same tendency occurs and the critical point is also at about $t_{1}=3.2$. (c), (g): The insulating phase at $U=6.0$. There is a visible single particle excitation gap around the Fermi energy. (d), (h): The density of states as a function of $\omega$ for different $U$ with fixed $t_{1}$.

inter-layer hopping $t_{1}$ for various interaction $U$ [see in Fig. 3], where $F$ is the free energy. We use $D_{A A}$ to describe the $D_{o c c}$ for $A_{1}$ and $A_{2}$ sites (hollow circles in Fig. 3), and $D_{B B}$ the $D_{o c c}$ for $B_{1}$ and $B_{2}$ sites (solid circles in Fig. 3). When $t_{1}=0, D_{o c c}$ decrease when $U$ is increased showing the localization is enforced by the interaction. When $t_{1} \neq t, D_{A A}$ is different with $D_{B B}$. It is found that when $t_{1}$ is increased, $D_{A A}$ increases while $D_{B B}$ decreases. This suggests the itinerancy of electrons in dimer sites is enhanced due to the increasing inter-layer hopping. However, be different with $D_{A A}, D_{B B}$ decreases while $t_{1}$ is increased. These results suggest the intra-hopping between dimer and non-dimer sites is weaken due to the forming of spinpolarized electrons in dimer sites.

We derive DOS from the imaginary time Green's function $G(\tau)$ to observe the single particle spectral for different interaction $U$ and $t_{1}$ by the maximum entropy method ${ }^{45,46}$. Fig. 4(a) and Fig. 4(e) shows the DOS for different inter-layer hopping when $U=2.5, T=0.1$ for A sites and B sites. It is found that, in weak interaction, the inter-layer hopping $t_{1}$ does not affect the metallic properties of BHL. In Fig. 4(b) and Fig. 4(f), we can find that the system keeps at a metallic state when $t_{1}=1.8$, and an obvious pseudo-gap is found when $t_{1}=2.2$. A metal-insulator transition happens when $t_{1}=3.2$ for $U=3.5$ and $T$ $=0.1$ for both $\mathrm{A}$ and $\mathrm{B}$ sites. When interaction being large, such as $U$ $=6.0$ [see Fig. 4(c) and Fig. 4(g)], the system stays at an insulating state, which is insensitive of $t_{1}$. Fig. 4(d) and Fig. 4(h) show the DOS for different $U$ when $t_{1}=1.0$ and $T=0.1$. An opened gap is found when the interaction is increased, which indicates a phase transition from metal to insulator. These results mean that the inter-layer hopping and Coulomb interaction play same roles for the metal-insulator phase transition.

In this report, the Fig. 5 shows the spectral function $A(k, \omega=0)$ near the Fermi surface for various $U$ and $t_{1} . A(k, \omega)$ is defined as:

$$
\begin{aligned}
& A(k, \omega) \approx-\frac{1}{\pi} \lim _{\omega \rightarrow 0} \operatorname{Im}_{k}(\omega+i \theta) \\
& G_{k}(i \omega)=\frac{1}{i \omega-\epsilon_{k}-\Sigma_{k}(i \omega)},
\end{aligned}
$$

where $i \omega$ is the Fermionic Matsubara frequency, $\epsilon_{k}$ denotes the dispersion relation, $\Sigma_{k}(i \omega)$ corresponds to the $k$-dependent self-energy, $k$ is the wave vector in the first Brillouin zone. Figs. 5(a)-(c) show the
$A(k, \omega=0)$ for different $t_{1}$ when $U=3.5$. When $t_{1}<3.2$, we can find that the peaks of $A(k, \omega=0)$ distribute in the $K$ and $K^{\prime}$ points [see Fig. 1(c)] in $k$-space, displaying a metallic behavior [see Figs. 5(a), (b)]. When $t_{1}=4.0$, the $A(k, \omega=0) \approx 0$ [see Fig. 5(c)], meaning that a gap can be found near the Fermi energy. This gapped behavior shows the system is an insulator. Similar behavior is found when $t_{1}=1.2$ and $U$ is increased [see Figs. 5(d) - (f)]. It means both spin-polarization and strongly correlated interaction can induce the localization of electrons, driving the system from metal to insulator.

The magnetic phase transition and a novel layer anti-ferromagnetic phase. In strongly correlated BHL, charge imbalance between the two sublattices sites causes different kinds of magnetic spontaneous symmetry breaking, dividing the sites into dimer sites and nondimer sites. In order to study the magnetic order, we use a magnetic order parameter defined as $m^{\alpha}=\frac{1}{N_{c}} \sum_{i_{\alpha}}^{N_{\alpha}}\left(<n_{i \uparrow}>-<n_{i \downarrow}>\right)$, where $\alpha$ denotes the sublattice $A_{1}, A_{2}, B_{1}$ and $B_{2}, i_{\alpha}$ is the lattice index for sublattice $\alpha$, and $N_{\alpha}$ means the site number for sublattice $\alpha$ [see in Fig. 1(a) and Fig. 1(b)]. Parameter $\left\langle n_{i \sigma}\right\rangle$ indicates the electron density in lattice site $i$ with spin index $\sigma$ (we set magnetization of $A_{1}$ as positive sign). Fig. 6 shows the evolution of $m^{\alpha}$ as a function of $t_{1}$ for $U=3.8$. It is found that when $U=3.8, m$ is zero for $t_{1}<1.0$, denoting that no magnetic order is formed for weak $t_{1}$. A magnetic state with anti-ferromagnetic order is found when $t_{1}>1.0$. When $t_{1}$ $>4.4$, the $m^{A_{1}}$ and $m^{A_{2}}$ decrease to zero, while $m^{B_{1}}, m^{B_{2}}$ are still finite, meaning a phase with non-magnetic order and magnetic order coexisting. This novel phase is called layer anti-ferromagnetic state, in which the dimer sites are non-magnetic and non-dimer sites are magnetic. The sketches of the possible magnetic order existing in BHL is shown in Figs. 7(a) - (c).

Finally, the phase diagram of magnetization about $t_{1}$ and $U$ for $T=$ 0.1 is shown in Fig. 7. A phase transition from paramagnet to antiferromagnet is found when $U$ is increased. A layer anti-ferromagnetic phase is found for large $t_{1}$, in which the magnetic of dimer sites keeps zero while non-dimer sites is nonzero. When $U>3.7$, a phase transition from layer anti-ferromagnetic state to paramagnetic state is found. 

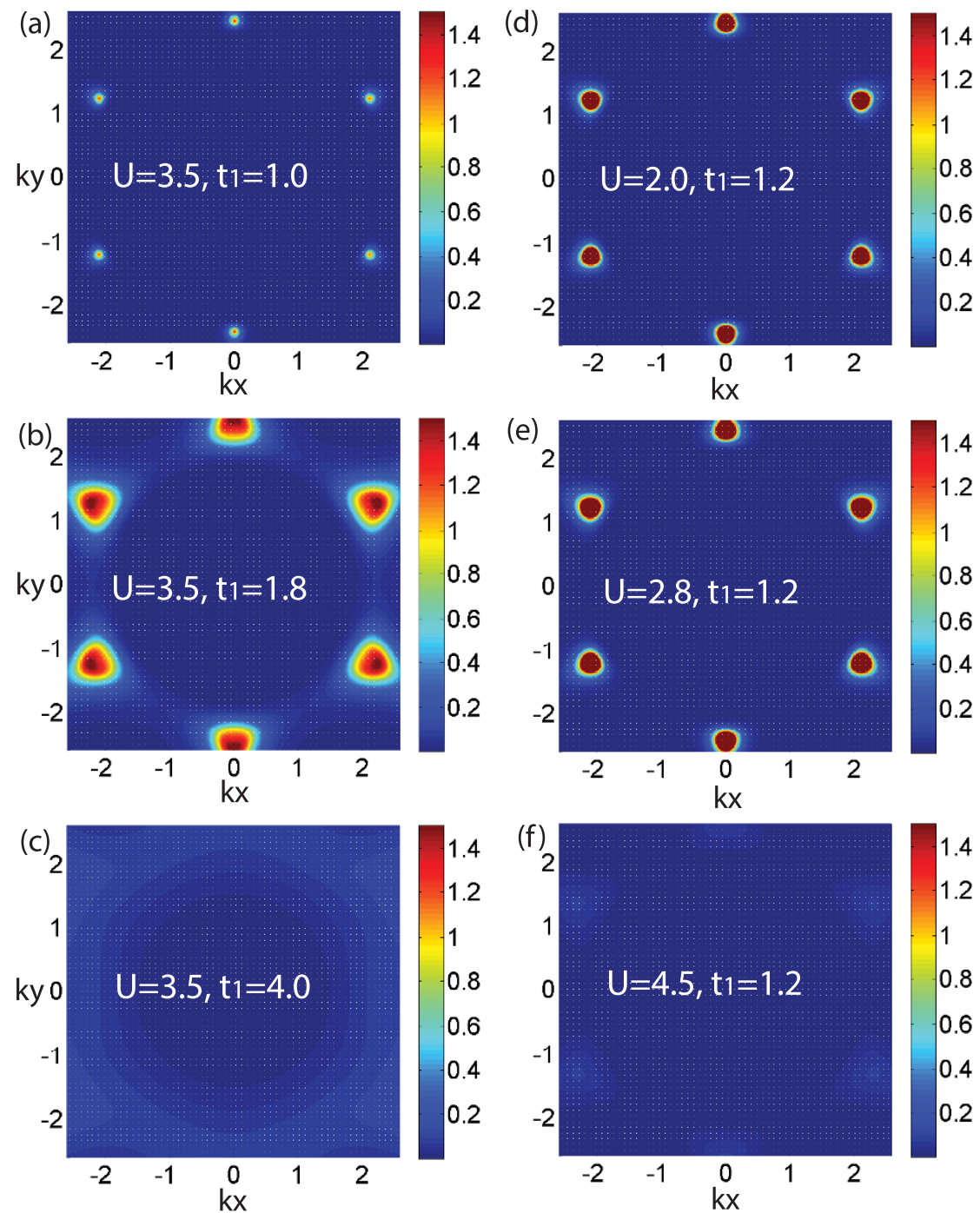

Figure 5 The evolution of spectral function near Fermi surface. The spectral function $A(k, \omega=0)$ near the Fermi surface for different inter-layer hopping $t_{1}$ at $T=0.1$ and $U=3.5$ : (a) $t_{1}=1.0$, (b) $t_{1}=1.8$, (c) $t_{1}=4.0$. The $A(k, \omega=0)$ when $T=0.1$ and $t_{1}=1.2$ for different interaction $U$ : (d) $U=2.0$, (e) $U=2.8$, (f) $U=4.5$.

Experimental protocol. We propose an experiment setup to investigate the phase transition in strongly correlated bilayer honeycomb lattice (BHL). The ${ }^{40} \mathrm{~K}$ atoms can be produced as a pure fermion condensate by evaporative cooling ${ }^{47}$, which provides two hyperfine states $\left|F, m_{F}\right\rangle=|9 / 2,-9 / 2\rangle \equiv|\uparrow\rangle$ and $\left|F, m_{F}\right\rangle=\mid 9 /$ $2,-7 / 2\rangle \equiv|\uparrow\rangle^{48}$. Three standing-wave laser beams are used to form the honeycomb lattice, and two extra laser beams along the $z$ direction suppress the tunneling between layers ${ }^{28}$. The potential of optical lattice is given by $V_{h}(x, y)=V_{0} \Sigma_{j=1,2,3} \sin ^{2}\left[k\left(x \cos \theta_{j}+y \sin \right.\right.$ $\left.\theta_{j}\right)+\pi / 2$ ], where $\theta_{1}=\pi / 3, \theta_{2}=2 \pi / 3, \theta_{3}=0$. Then, we use another three standing-wave laser beams with a $2 \pi / 3$ angle between each other to form triangular lattice. The potential is given by $V_{t}(x, y)=V_{0}\left[3+4 \cos \left(k_{x} x / 2\right) \cos \left(\sqrt{3} k_{y} y / 2\right)+2 \cos \left(\sqrt{3} k_{y} y\right)\right] \cdot k_{x}$ and $k_{y}$ are the two components of the wave vector $k=2 \pi / \lambda$ in these two types of lattices, where $\lambda=738 \mathrm{~nm}$ is wavelength of the laser, and $V_{0}$ is given in recoil energy $E_{r}=\hbar^{2} k^{2} / 2 m$. Inserting the triangular lattice between two layers of honeycomb lattice, the Bernal stacking BHL with trapped ${ }^{40} \mathrm{~K}$ atoms can be formed ${ }^{49,50}$. In BHL the intra-layer hopping $t=(4 / \sqrt{\pi}) E_{r}^{1 / 4} V_{0}^{3 / 4} \exp \left[-2\left(V_{0} / E_{r}\right)^{1 / 2}\right]$ is adjusted by the periodic potential of laser beam and $t_{1}$ can be tuned by changing the wavelength of laser beam in $z$ direction. The on-site interaction $U=\sqrt{8 / \pi} k a_{s} E_{r}\left(V_{0} / E_{r}\right)^{3 / 4}$ is determined by the s-wave scattering length $a_{s}$, which can be tuned by Feshbach resonance. The temperature can be extracted from the time-of-flight images ${ }^{51}$.

It should be mentioned that, we can detect the numbers of double occupied sites to confirm whether the metal-Mott insulator transition happens. Firstly, we have to increase the depth of the optical lattice to prevent further tunneling of atoms. Next, the energy of the atoms on doubly occupied sites is shifted by approaching a Feshbach resonance. Then the one spin component of atoms on double occupied sites is transferred to a new magnetic sublevel by radiofrequency pulse method. Finally, the double occupancy can be deduced by the absorption images ${ }^{52,53}$.

To get Fermi surface in experimental, we ramp down the optical lattice slowly enough first, and the atoms stay adiabatically in the lowest band while quasi-momentum is approximately conserved. Then the lattice potential is lowered to zero rapidly, by switching off the confining potential and the atoms can expand ballistically for several milliseconds. The Fermi surface can be obtained by a absorption image $e^{54,55}$.

\section{Discussion}

In this work, we have investigated the metal-insulator transition and magnetic phase transition in strongly correlated bilayer honeycomb lattice using cellular dynamical mean-field theory (CDMFT) 


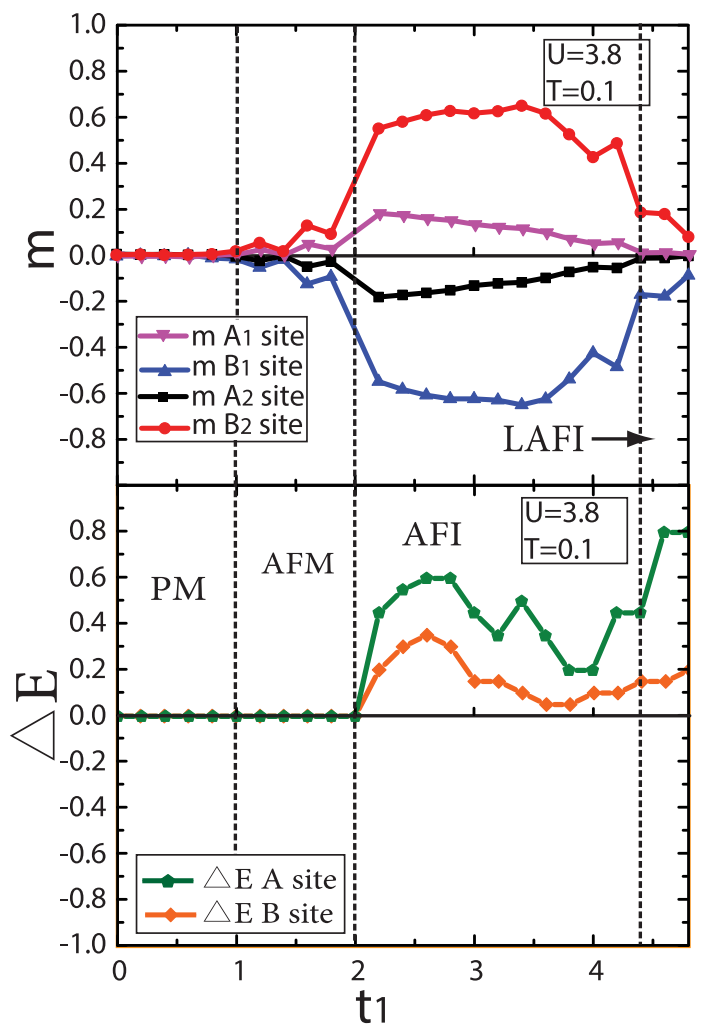

Figure $6 \mid$ The evolution of the magnetic order parameter $m$. The evolution of magnetic order parameter $m$ at $T=0.1$ and $U=3.8$. A paramagnetic phase is found in weak $t_{1}$. For $t_{1}>1.0$, the magnetic parameter $m$ is nonzero and has opposite sign between $A_{1} / A_{2}$ sites and $B_{1} /$ $B_{2}$ sites. The system goes into anti-ferromagnetic phase. At large $t_{1}$ the magnetization of $A_{1} / A_{2}$ sites are more easily decreasing to zero while $B_{1} / B_{2}$ sites are still nonzero. The system is layer anti-ferromagnetic phase. Single particle excitation gap $\Delta \mathrm{E}$ denoted by the dark green solid line and orange solid line, divide the phase into paramagnetic metal (PM), anti-

ferromagnetic metal (AFM), anti-ferromagnetic insulator (AFI) and layer anti-ferromagnetic insulator (LAFI).

combining with continue-time quantum Monte Carlo (CTQMC) method. In lower temperature case we map the phase diagram as a function of interaction $U$, inter-layer hopping $t_{1}$ and magnetization $m$. It shows that the inter-layer hopping affects the electrons to form spin-polarized electrons, and an insulating state is induced. A layer anti-ferromagnetic phase is found at large $t_{1}$, in which the magnetization of dimer sites is zero while non-dimer keeps finite value. Therefore, the inter-layer hopping $t_{1}$ plays an important role to form a singular magnetic spontaneous symmetry breaking phase. Our study may provide a helpful step for understanding the interaction and inter-layer hopping driven metal-insulator transition, the exotic magnetic order with asymmetry breaking and the possible magneticnonmagnetic order coexisting state.

\section{Methods}

The cellular dynamical mean-field theory. We combine the cellular dynamical mean-field theory (CDMFT) with continuous time quantum Monte Carlo (CTQMC) method to determine the metal-insulator transition and magnetic phase transition in the strongly correlated bilayer honeycomb lattice. In low-dimensional systems, quantum fluctuations are much stronger than the higher dimensions. The nonlocal effect is much important in this case. Dynamical mean-field theory ignoring the nonlocal correlations leads lots of errors in calculation. Therefore, we use CDMFT, as the advanced method in our work. We map the original lattice onto a 12 -site effective cluster embedded in a self-consistent bath field [see Fig. 1(b)]. Starting with a guessing self-energy $\Sigma(i \omega)$ (which is independent of momentum ${ }^{56}$ ), we can get the Weiss field $G_{0}(i \omega)$ obtained by the coarse-grained Dyson equation:

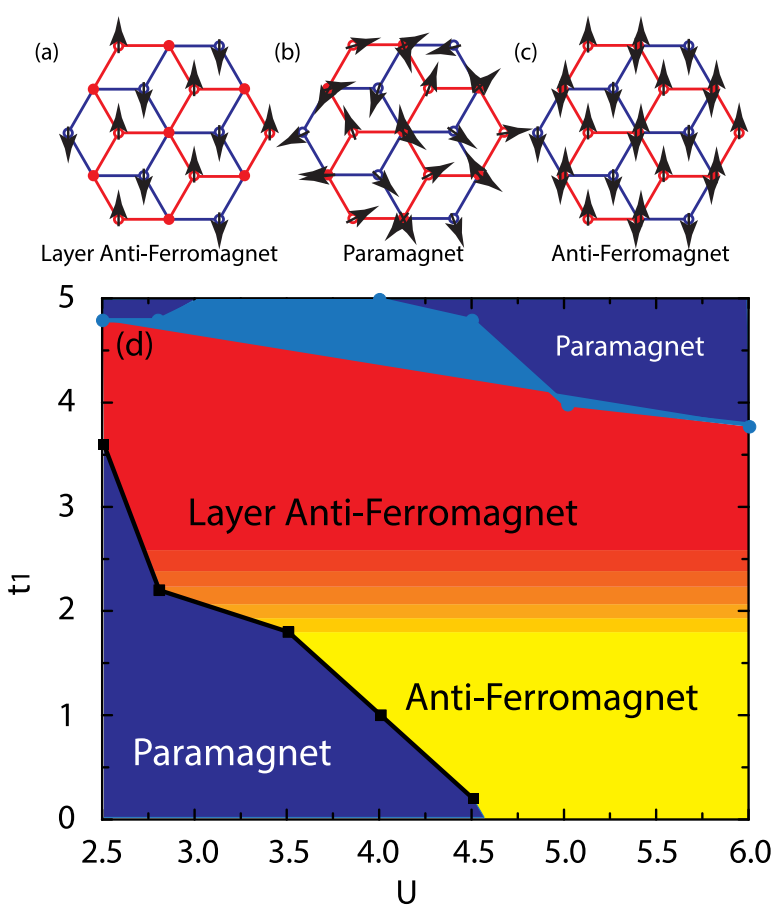

Figure $7 \mid$ The phase diagram of magnetic phase transition. In weak interaction $U$ and weak inter-layer hopping $t_{1}$, the system is paramagnet. When we increase $U$, the system undergoes a magnetic phase transition to anti-ferromagnet. When we increase $t_{1}$, the magnetization of $A_{1} / A_{2}$ sites decrease to zero while $B_{1} / B_{2}$ sites stay finite. The system becomes layer antiferromagnet. In the region where $t_{1}>5.0$, the system returns to paramagnetic phase.

$$
G_{0}^{-1}(i \omega)=\left(\sum_{k} \frac{1}{i \omega-t(k)-\Sigma(i \omega)}\right)^{-1}+\Sigma(i \omega),
$$

where $i \omega$ is Fermionic Matsubara frequency, $\mu$ is the chemical potential, $k$ is in the reduced Brillouin zone of the super-lattice, and $t(k)$ is hopping matrix for the superlattice. The form of $t(k)$ is:

$$
t(k)=\left\{\begin{array}{llllllllllll}
0 & t & 0 & t \delta_{1} & 0 & t & 0 & 0 & 0 & t_{1} \delta_{1} & 0 & 0 \\
t & 0 & t & 0 & t \delta_{2} & 0 & 0 & 0 & 0 & 0 & 0 & 0 \\
0 & t & 0 & t & 0 & t \delta_{3} & 0 & t_{1} & 0 & 0 & 0 & 0 \\
t \delta_{1}^{*} & 0 & t & 0 & t & 0 & 0 & 0 & 0 & 0 & 0 & 0 \\
0 & t \delta_{2}^{*} & 0 & t & 0 & t & 0 & 0 & 0 & 0 & 0 & t_{1} \\
t & 0 & t \delta_{3}^{*} & 0 & t & 0 & 0 & 0 & 0 & 0 & 0 & 0 \\
0 & 0 & 0 & 0 & 0 & 0 & 0 & t & 0 & t \delta_{1} & 0 & t \\
0 & 0 & t_{1} & 0 & 0 & 0 & t & 0 & t & 0 & t \delta_{2} & 0 \\
0 & 0 & 0 & 0 & 0 & 0 & 0 & t & 0 & t & 0 & t \delta_{3} \\
t_{1} \delta_{1}^{*} & 0 & 0 & 0 & 0 & 0 & t \delta_{1}^{*} & 0 & t & 0 & t & 0 \\
0 & 0 & 0 & 0 & 0 & 0 & 0 & t \delta_{2}^{*} & 0 & t & 0 & t \\
0 & 0 & 0 & 0 & t_{1} & 0 & t & 0 & t \delta_{3}^{*} & 0 & t & 0
\end{array}\right\}
$$

where $\delta_{1}=e^{i \mathbf{k} \mathbf{a}_{1}}, \delta_{2}=e^{i \mathbf{k} \cdot\left(\mathbf{a}_{1}-\mathbf{a}_{2}\right)}, \delta_{3}=e^{-i \mathbf{k}^{\prime} \mathbf{a}_{2}}$ and $\mathbf{a}_{1}, \mathbf{a}_{2}$ are real lattice vectors as shown in Fig. 1(a). The cluster Green's function $G(i \omega)$ can be gotten by the impurity solver. In our work, we use the numerically exact CTQMC simulation as impurity solver and take $5 \times 10^{6}$ QMC sweeps for each CDMFT loop ${ }^{42}$. The new self-energy $\Sigma(i \omega)$ is recalculated by the Dyson equation:

$$
\Sigma(i \omega)=G_{0}^{-1}(i \omega)-G^{-1}(i \omega) .
$$

This iterative loop repeated until self-energy is converged.

The CTQMC method as impurity solver can be taken as follows. We start the procedure at partition function, which can be written as:

$$
Z=T_{r} e^{-\beta H}=Z_{0} T_{\tau}\left[\sum_{k} \frac{1}{k !}\left(-\int_{0}^{\beta} H_{1}(\tau) d \tau\right)^{k}\right],
$$


where $T_{\tau}$ is time-ordering operator, $H_{1}(\tau)=e^{\tau H_{0}} H_{1} e^{-\tau H_{0}}$ is $H_{1}$ in the interaction picture, and $Z_{0}=T_{r} e^{-\beta H_{0}}$ is a partition function for the unperturbed term. Putting $H_{1}$ $=U \Sigma_{i} n_{i \downarrow} n_{i \uparrow}$ in Eq. 4, the partition function is

$Z=Z_{0} \sum_{k} \frac{(-U)^{k}}{k !} \int \cdots \int d r_{1} \cdots d r_{k}\left\langle T_{\tau} n_{\uparrow}\left(r_{1}\right) \cdots n_{\uparrow}\left(r_{k}\right)\right\rangle_{0}\left\langle T_{\tau} n_{\downarrow}\left(r_{1}\right) \cdots n_{\downarrow}\left(r_{k}\right)\right\rangle_{0}$.

Here \langle\rangle$_{0}$ indicates a theromdynamic average with respect to $e^{-\beta H_{0}}$. Using Wick's theorem, for each order in $k,\left\langle T_{\tau} n_{\sigma}\left(r_{1}\right) \cdots n_{\sigma}\left(r_{k}\right)\right\rangle_{0}(\sigma=\uparrow, \downarrow)$ can be written as determinant $\operatorname{det} D(k)$ :

$$
D(k)=\left\{\begin{array}{cccc}
G^{0}\left(r_{1}, r_{1}\right) & G^{0}\left(r_{1}, r_{2}\right) & \cdots & G^{0}\left(r_{1}, r_{k}\right) \\
G^{0}\left(r_{2}, r_{1}\right) & G^{0}\left(r_{2}, r_{2}\right) & \cdots & G^{0}\left(r_{2}, r_{k}\right) \\
\cdot & \cdot & \cdot & \cdot \\
\cdot & \cdot & \cdot & \cdot \\
G^{0}\left(r_{k}, r_{1}\right) & G^{0}\left(r_{k}, r_{2}\right) & \cdots & G^{0}\left(r_{k}, r_{k}\right)
\end{array}\right\},
$$

where $G^{0}$ is non-interacting Green's function. There is no spin index in $D(k)$ for the determinants of spin-un and -down being equivalent. Like classical Monte Carlo, by integrand of Eq. 5, we can get the weight of order $k$

$$
W_{k}=(-\delta \tau U)^{k} \operatorname{det} D_{\uparrow}(k) \operatorname{det} D_{\downarrow}(k),
$$

where $\delta \tau=\beta / L$ is slice of imaginary time. We can get the standard Metropolis acceptance ratio $R$ of adding vertex by the detailed balance condition:

$$
\begin{gathered}
\frac{1}{L \cdot N} W_{k} P_{k \rightarrow k+1}=\frac{1}{k+1} W_{k+1} P_{k+1 \rightarrow k}, \\
R=\frac{P_{k \rightarrow k+1}}{P_{k+1 \rightarrow k}}=-\frac{U \beta N}{k+1}\left(\frac{\operatorname{det} D_{\uparrow}(k+1) \operatorname{det} D_{\downarrow}(k+1)}{\operatorname{det} D_{\uparrow}(k) \operatorname{det} D_{\downarrow}(k)}\right) .
\end{gathered}
$$

Here $P_{k \rightarrow k+1}$ is the probability to increase the order from $k$ to $k+1\left(P_{k+1 \rightarrow k}\right.$ the probability to decrease the order from $k+1$ to $k$ ), $\frac{1}{L \cdot N}$ is probability to choose a position in time and space for vertex you intend to add while $\frac{1}{k+1}$ is the probability to choose one vertex you intend to remove of from the existing $k+1$ noes. To calculate the ratio $R$, we have to deal with the function $\operatorname{det} D(k+1) / \operatorname{det} D(k)$.

$$
\operatorname{det} D(k+1) / \operatorname{det} D(k)=\operatorname{det}(I+(D(k+1)-D(k)) M(k))=\lambda,
$$

$M_{\sigma}(k)=D_{\sigma}^{-1}(k)$, we can easily get the value of $\lambda$ in matrix form:

$$
\begin{aligned}
\operatorname{det}\left(\begin{array}{ccccc}
1 & 0 & \cdots & 0 & G^{0}\left(r_{1}, r_{k+1}\right) \\
0 & 1 & \cdots & 0 & G^{0}\left(r_{2}, r_{k+1}\right) \\
\cdot & \cdot & \ldots & \cdot & \cdot \\
\cdot & \cdot & \cdots & \cdot & \cdot \\
0 & 0 & \cdots & 1 & G^{0}\left(r_{k}, r_{k+1}\right) \\
G^{0}\left(r_{k+1}, r_{i}\right) M(k)_{i, 1} & G^{0}\left(r_{k+1}, r_{i}\right) M(k)_{i, 2} & \cdots & G^{0}\left(r_{k+1}, r_{i}\right) M(k)_{i, k} & G^{0}\left(r_{k+1}, r_{k+1}\right)
\end{array}\right) \\
=G^{0}\left(r_{k+1}, r_{K+1}\right)-G^{0}\left(r_{k+1}, r_{i}\right) M(k)_{i, j} G^{0}\left(r_{j}, r_{k+1}\right)=\lambda .
\end{aligned}
$$

Then it is easy to obtain the update $M$ for the order $k+1$ by numerical method:

$$
M(k+1)=\left(\begin{array}{cccc}
\cdot & \cdot & \cdot & -L_{1, k+1} \lambda^{-1} \\
\cdot & M_{i, j}^{\prime} & \cdot & -L_{2, k+1} \lambda^{-1} \\
\cdot & \cdot & \cdot & -L_{k, k+1} \lambda^{-1} \\
\cdot & \cdot & \cdot & \cdot \\
-\lambda^{-1} R_{k+1,1} & -\lambda^{-1} R_{k+1,2} & \cdots & -\lambda^{-1}
\end{array}\right),
$$

where the factor of the matrix is $M_{i, j}^{\prime}=M(k)_{i, j}+L_{i, k+1} \lambda^{-1} R_{k+1, j}, R_{i, j}=G^{0}(i, l) M(k)_{l, j}$ and $L_{i, j}=M(k)_{i, l} G^{0}(l, j)$. For the step $k-1$, we can also get the radio $R$ and update formulas of $M(k-1)$ :

$$
\begin{gathered}
R=\frac{k}{U \beta N}\left(\frac{\operatorname{det} D_{\uparrow}(k-1) \operatorname{det} D_{\downarrow}(k-1)}{\operatorname{det} D_{\uparrow}(k) \operatorname{det} D_{\downarrow}(k)}\right), \\
M_{i, j}(k-1)-M_{i, j}(k)-M_{i, l}(k) M_{l, j}(k) / M_{l, l}(k) .
\end{gathered}
$$

Using the update formula for $M$, the Green's function can be obtained both in imaginary time and at Matsubara frequencies:

$$
\begin{aligned}
& G\left(\tau-\tau^{\prime}\right)=G^{0}\left(\tau-\tau^{\prime}\right)-G^{0}\left(\tau-\tau_{i}\right) M_{i, j} G^{0}\left(\tau_{j}-\tau^{\prime}\right), \\
& G(i \omega)=G_{0}(i \omega)-G_{0}(i \omega)\left[\frac{1}{\beta} \sum_{i, j} M_{i, j} e^{-\omega\left(\tau_{i}-\tau_{j}\right)}\right] G_{0}(i \omega) .
\end{aligned}
$$

Here $G_{0}(i \omega)$ is a bare Green's function.

1. Kharitonov, M. Canted Antiferromagnetic Phase of $v=0$ Quantum Hall State in Bilayer Graphene. Phys. Rev. Lett. 109, 046803 (2012).

2. Zhang, Y. Y. et al. Quantum Blockade and Loop Currents in Graphene with Topological Defects. Phys. Rev. B 78, 155413 (2008).

3. Novoselov, K. S. et al. Unconventional Quantum Hall Effect and Berry's Phase of $2 \pi$ in Bilayer Graphene. Nature Phys. 2, 177 (2006).

4. Freitag, F., Trbovic, J., Weiss, M. \& Shönenberger, C. Spontaneously Gapped Ground State in Suspended Bilayer Graphene. Phys. Rev. Lett. 108, 076602 (2012).

5. Feldman, B. E., Martin, J. \& Yacoby, A. Broken-Symmetry States and Divergent Resistance in Suspended Bilayer Graphene. Nature Phys. 5, 889 (2009).

6. Zhao, Y., Cadden-Zimansky, P., Jiang, Z. \& Kim, P. Symmetry Breaking in the Zero-Energy Landau Level in Bilayer Graphene. Phys. Rev. Lett. 104, 066801 (2010).

7. Weitz, R. T., Allen, M. T., Feldman, B. E., Martin, J. \& Yacoby, A. BrokenSymmetry Sates in Doubly Gated Suspended Bilayer Graphene. Science 330, 812 (2010).

8. Maher, P. et al. Evidence for a Spin Phase Transition at Charge Neutrality in Bilayer Graphene. Nature Phys. 9, 154 (2013).

9. Kane, C. L. \& Mele, E. J. $Z_{2}$ Topological Order and Quantum Spin Hall Effect. Phys. Rev. Lett. 95, 146802 (2005).

10. Hosseini, M. V. \& Zareyan, M. Model of an Exotic Chiral Superconducting Phase in a Graphene Bilayer. Phys. Rev. Lett. 108, 147001 (2012).

11. Yan, X. Z. Superconductivity in the Quasi-Two-Dimensional Hubbard Model. Phys. Rev. B 71, 104520 (2005).

12. Zhang, Y. Y. et al. Localization and the Kosterlitz-Thouless Transition in Disorderd Graphene. Phys. Rev. Lett. 102, 106401 (2009).

13. Mezzacapo, F. \& Boninsegni, M. Ground-State Phase Diagram of the Quantum $J_{1}$ - $J_{2}$ model on the Honeycomb Lattice. Phys. Rev. B 85, 060402 (2012).

14. Anderson, P. W. The Resonating Valence Bond State in $\mathrm{La}_{2} \mathrm{CuO}$ and Superconductivity. Science 235, 1196 (1987).

15. Wang, M. et al. Antiferromagnetic Order and Superlattice Structure in Nonsuperconducting and Superconducting $R b_{y} F e_{1.6+} S e_{2}$. Phys. Rev. B 84, 094504 (2011).

16. Wu, W., Chen, Y. H., Tao, H. S., Tong, N. H. \& Liu, W. M. Interacting Dirac Fermions on Honeycomb Lattice. Phys. Rev. B 82, 245102 (2010).

17. Vafek, O. Interacting Fermions on the Honeycomb Bilayer: From Weak to Strong Coupling. Phys. Rev. B 82, 205106 (2010).

18. McCann, E. \& Koshino, M. The Electronic Properties of Bilayer Graphene. Rep. Prog. Phys. 76, 056503 (2013).

19. Nilsson, J., Castro Neto, A. H., Peres, N. M. R. \& Guinea, F. Electron-Electron Interactions and the Phase Diagram of Graphene Bilayer. Phys. Rev. B 73, 214418 (2006).

20. McCann, E. Asymmetry Gap in the Electronic Band Structure of Bilayer Graphene. Phys. Rev. B 74, 161403 (2006).

21. Kechedzhi, K., Fal'ko, V. I., McCann, E. \& Altshuler, B. L. Influence of Trigonal Warping on Interference Effect in Bilayer Graphene. Phys. Rev. Lett. 98, 176806 (2007).

22. Nilsson, J., Castro Neto, A. H., Guinea, F. \& Peres, N. M. R. Electronic Properties of Graphene Multilayers. Phys. Rev. Lett. 97, 266801 (2006).

23. Lopes dos Santos, J. M. B., Peres, N. M. R. \& Castro Neto, A. H. Graphene Bilayer with a Twist: Electronic Structure. Phys. Rev. Lett. 99, 256802 (2007).

24. Abergel, D. S. L. \& Chakraborty, T. Long-Range Coulomb Interaction in Bilayer Graphene. Phys. Rev. Lett. 102, 056807 (2009).

25. Jaksch, D., Bruder, C., Cirac, J. I., Gardiner, C. W. \& Zoller, P. Cold Bosonic Atoms in Optical Lattices. Phys. Rev. Lett. 81, 3108 (1998).

26. Hofstetter, W., Cirac, J. I., Zoller, P., Demler, E. \& Lukin, M. D. High-Temperature Superfluidity of Fermionic Atoms in Optical Lattices. Phys. Rev. Lett. 89, 220407 (2002).

27. Greiner, M., Mandel, O., Esslinger, T., Hänsch, T. W. \& Bloch, I. Quantum Phase Transition from a Superfluid to a Mott Insulator in a Gas of Ultracold Atoms. Nature 415, 39 (2002).

28. Duan, L. M., Demler, E. \& Lukin, M. D. Controlling Spin Exchange Interactions of Ultracold Atoms in Optical Lattices. Phys. Rev. Lett. 91, 090402 (2003).

29. Soltan-Panahi, P. et al. Multi-Component Quantum Gases in Spin-Dependent Hexagonal Lattices. Nature Phys. 7, 434 (2011).

30. Gemelke, N., Zhang, X., Hung, C.-L. \& Chin, C. In Situ Observation of Incompressible Mott-Insulating Domains in Ultracold Atomic Gases. Nature 460, 995 (2009).

31. Chen, Y. H., Tao, H. S., Yao, D. X. \& Liu, W. M. Kondo Metal and Ferrimagnetic Insulator on the Triangular Kagome Lattice. Phys. Rev. Lett. 108, 246402 (2012).

32. Metzner, W. \& Vollhardt, D. Correlated Lattice Fermions $d=\infty$ Dimensions. Phys. Rev. Lett. 62, 324 (1989). 
33. Georges, A. \& Kotliar, G. Hubbard Model in Infinite Dimensions. Phys. Rev. B 45, 6479 (1992).

34. Bulla, R. Zero Temperature Metal-Insulator Transition in the InfiniteDimensional Hubbard Model. Phys. Rev. Lett. 83, 136 (1999).

35. Georges, A. \& Kotliar, G. Dynamical Mean-Field Theory of Strongly Correlated Fermion Systems and the Limit of Infinite Dimensions. Rev. Mod. Phys. 68, 13 (1996).

36. Kotliar, G., Savrasov, S. Y., Pálsson, G. \& Biroli, G. Cellular Dynamical Mean Field Approach to Strongly Correlated Systems. Phys. Rev. Lett. 87, 186401 (2001)

37. Maier, T., Jarrell, M., Pruschke, T. \& Hettler, M. H. Quantum Cluster Theories. Rev. Mod. Phys. 77, 1027 (2005).

38. Tong, N. H. Extended Variational Cluster Approximation for Correlated Systems. Phys. Rev. B 72, 115104 (2005).

39. Liu, H. D., Chen, Y. H., Lin, H. F., Tao, H. S. \& Liu, W. M. Antiferromagnetic Metal and Mott Transition on Shastry-Sutherland Lattice. Scientific Reports 4, 4829 (2014).

40. Bolech, C. J., Kancharla, S. S. \& Kotliar, G. Cellular Dynamical Mean-Field Theory for the One-Dimensional Extended Hubbard Model. Phys. Rev. B 67, 075110 (2003).

41. Cai, Z., Hung, H. H., Wang, L. \& Wu, C. J. Quantum Magnetic Properties of the $S$ $U(2 N)$ Hubbard Model in the Square Lattice: A Quantum Monte Carlo Study. Phys. Rev. B 88, 125108 (2013).

42. Rubtsov, A. N., Savkin, V. V. \& Lichtenstein, A. I. Continuous-Time Quantum Monte Carlo Method for Fermions. Phys. Rev. B 72, 035122 (2005).

43. Zhu, S. L., Wang, B. \& Duan, L. M. Simulation and Detection of Dirac Fermions with Cold Atoms in an Optical Lattice. Phys. Rev. Lett. 98, 260402 (2007).

44. Kancharla, S. S. \& Okamoto, S. Band Insulator to Mott Insulator Transition in a Bilayer Hubbard Model. Phys. Rev. B 75, 193103 (2007).

45. Hu, H., Jiang, L., Liu, X. J. \& Pu, H. Probing Anisotropic Superfluidity in Atomic Fermi Gases with Rashba Spin-Orbit Coupling. Phys. Rev. Lett. 107, 195304 (2011).

46. Jarrell, M. \& Gubernatis, J. E. Bayesian Inference and the Analytic Continuation of Imaginary-Time Quantum Monte Carlo Data. Phys. Rep. 269, 133 (1996).

47. O’Hara, K. M., Hemmer, S. L., Gehm, M. E., Granade, S. R. \& Thomas, J. E Observation of a Strongly Interacting Degenerate Fermi Gas of Atoms. Science 298, 2179 (2002)

48. Hackermüller, L. et al. Anomalous Expansion of Attractively Interacting Fermionic Atoms in an Optical Lattice. Science 327, 1621 (2010).

49. Tung, S., Schweikhard, V. \& Cornell, E. A. Observation of Vortex Pinning in BoseEinstein Condensates. Phys. Rev. Lett. 97, 240402 (2006).

50. Hou, J. M. Energy Bands and Landau Levels of Ultracold Fermions in the Bilayer Honeycomb Optical Lattice. J. Mod. Opt. 56, 1182 (2009).
51. Schneider, U. et al. Metallic and Insulating Phases of Repulsively Interacting Fermions in a 3D Optical Lattice. Science 322, 1520 (2009).

52. Jördens, R., Strohmaier, N., Günter, K., Moritz, H. \& Esslinger, T. A Mott Insulator of Fermionic Atoms in an Optical Lattice. Nature 455, 204(2008).

53. Stöferle, T., Moritz, H., Günter, K., Köhl, M. \& Esslinger, T. Molecules of Fermionic Atoms in an Optical Lattice. Phys. Rev. Lett. 96, 030401 (2006).

54. Köhl, M., Moritz, H., Stöferle, T., Günter, K. \& Esslinger, T. Fermionic Atoms in a Three Dimensional Optical Lattice: Observing Fermi Surface, Dynamics, and Interactions. Phys. Rev. Lett. 94, 080403 (2005).

55. Chin, J. K. et al. Evidence for Superfluidity of Ultracold Fermions in an Optical Lattice. Nature 443, 961 (2006).

56. Müller-Hartmann, E. Correlated Fermions on a Lattice in High Dimensions. Z. Phys. B 74, 507 (1989)

\section{Acknowledgments}

This work was supported by the NKBRSFC under grants Nos. 2011CB921502, 2012CB821305, NSFC under grants Nos. 61227902, 61378017, and SPRPCAS under grants No. XDB01020300.

\section{Author contributions}

H.S.T. performed calculations. H.S.T., Y.H.C., H.D.L., H.F.L., W.M.L. analyzed numerical results. H.S.T., Y.H.C., W.M.L. contributed in completing the paper.

\section{Additional information}

Competing financial interests: The authors declare no competing financial interests. How to cite this article: Tao, H.-S., Chen, Y.-H., Lin, H.-F., Di Liu, H.- \& Liu, W.-M. Layer Anti-Ferromagnetism on Bilayer Honeycomb Lattice. Sci. Rep. 4, 5367; DOI:10.1038/ srep05367 (2014).

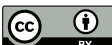

This work is licensed under a Creative Commons Attribution 4.0 International License. The images or other third party material in this article are included in the article's Creative Commons license, unless indicated otherwise in the credit line; if the material is not included under the Creative Commons license, users will need to obtain permission from the license holder in order to reproduce the material. To view a copy of this license, visit http://creativecommons.org/licenses/by/4.0/ 